\title{
$\gamma$-射线辐照对 $\mathrm{MSO}_{4}: \mathrm{Sm}^{3+}$ 发光体的影响 ${ }^{*}$
}

\author{
巩雄 张桂兰汤国庆陈文驹 \\ (南开大学现代光学研究所, 天津 300071) \\ 杨宏秀
}

(天津大学化学系, 天津 300072)

\section{关键词碱土金属硫酸盐 钐离子 $\gamma$ 辐照}

稀土掺杂的碱土金属硫酸盐是一类重要的发光材料 [1]. 钐掺杂的发光材料可望用作光谱 烧孔材料和光存储材料 [2, 3]. 实验证明, 含掺杂离子的晶体被辐照后, 容易产生缺陷, 形成色 心; 同时, 晶体内掺杂离子又可作为陷阱, 俘获由辐照产生的电子或空穴, 使掺杂离子的价态发 生改变. 国内外对铈掺杂的 $\mathrm{CaF}_{2}$ 晶体发光机理进行了一定研究 ${ }^{[4,5]}$, 但未见对钐掺杂的碱土金 属硫酸盐发光体的研究报道. 本文利用 ${ }^{60} \mathrm{Co} \gamma$ 射线对 $\mathrm{MSO}_{4}: x \mathrm{Sm}^{3+}(\mathrm{M}=\mathrm{Ca}, \mathrm{Sr}, \mathrm{Ba}$, $x=0.001 \sim 0.005)$ 发光体进行了辐照, 研究了辐照前后发光体的晶体结构, 光谱特征变化. 发 现发光体被 $\gamma$ 射线辐照后, 其晶形未变, 晶胞参数变小; 在同一发光体中同时观察到了 $\mathrm{Sm}^{3+}$, $\mathrm{Sm}^{2+}$ 两种离子的发射光谱. 这将有益于探索新型发光材料.

\section{1 实验}

根据化学计量关系, 采取共沉淀并加法制得初产物, 初产物移至刚玉坩埚中, 于 $150{ }^{\circ} \mathrm{C}$ 下 焙烧 $10 \mathrm{~h}$, 即得所需粉样 $\mathrm{MSO}_{4}: x \mathrm{Sm}^{3+}(\mathrm{M}=\mathrm{Ca}, \mathrm{Sr}, \mathrm{Ba} . x=0.001,0.002,0.003,0.004,0.005)$.

以 ${ }^{60} \mathrm{Co}$ 为放射源, 在剂量 $7.04 \times 10^{5} \mathrm{rad}$ 条件下对粉样进行 $\gamma$-射线辐照.

粉样用日本理学 D/MAX-IIIC 型 X-射线粉末衍射仪测定结构, 采用 TREOR 程序计算有 关参数. 激发和发射光谱在日本岛津 FL-540 型苂光分光光度计上测定.

\section{2 结果与讨论}

\section{$2.1 \mathrm{MSO}_{4}: x \mathrm{Sm}^{3+}$ 晶体结构和晶粒大小}

$\gamma$ - 射线辐照前后发光体 $\mathrm{MSO}_{4}: x \mathrm{Sm}^{3+}(\mathrm{M}=\mathrm{Ca}, \mathrm{Sr}, \mathrm{Ba}, x=0.001 \sim 0.005) \mathrm{XRD}$ 分析和 TREOR 程序计算结果表明, 所有粉样均为单一物相, 属于正交晶系, 空间群分别为 $B_{\mathrm{mm}}$ $\left(\mathrm{CaSO}_{4}: \mathrm{Sm}^{3+}\right), P_{\mathrm{nma}}\left(\mathrm{SrSO}_{4}: \mathrm{Sm}^{3+}\right), P_{\mathrm{bmm}}\left(\mathrm{BaSO}_{4}: \mathrm{Sm}^{3+}\right)$. 晶胞参数 $((\mathrm{a}) 、(\mathrm{~b}) 、(\mathrm{c}))$ 与钐掺杂量 $(x)$ 关 系如图 1 所示. 由图可知, 晶胞参数随钐掺杂量增大而线性增大, 辐照使晶胞参数相应变小. 这是 由于 $\mathrm{M}^{2+}(\mathrm{Ca}, \mathrm{Sr}, \mathrm{Ba})$ 离子半径较 $\mathrm{Sm}^{3+}$ 离子半径大, $\gamma$ 射线辐照后产生一定数目的电子所致 ${ }^{\left[{ }^{2}\right.}$.

1995-01-19 收稿, 1995-06-06 收修改稿

* 国家教委博士点基金资助项目 

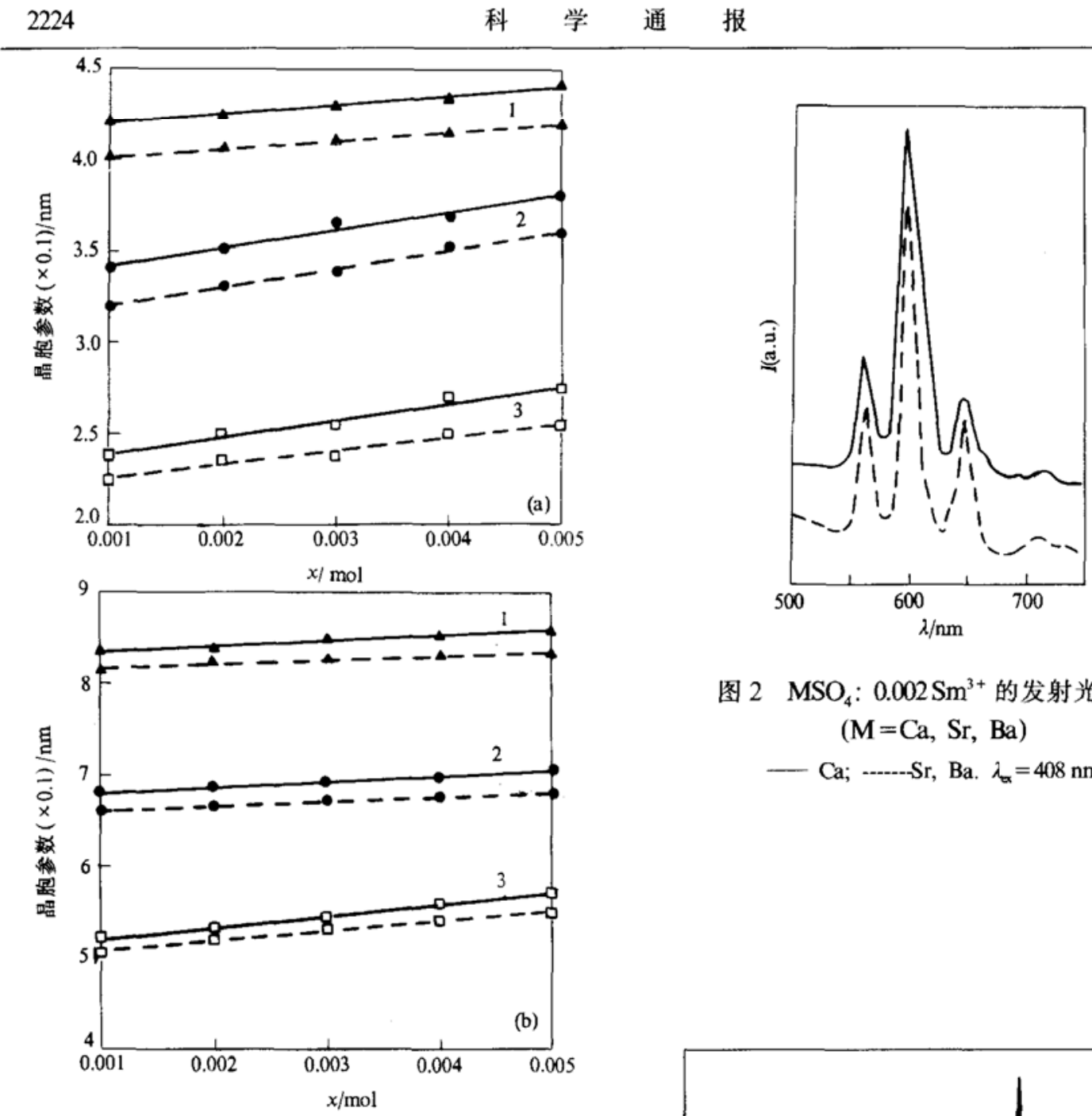

图 $2 \mathrm{MSO}_{4}: 0.002 \mathrm{Sm}^{3+}$ 的发射光谱 $(\mathrm{M}=\mathrm{Ca}, \mathrm{Sr}, \mathrm{Ba})$

- Ca; -..--.sr, Ba. $\lambda_{\alpha}=408 \mathrm{~nm}$

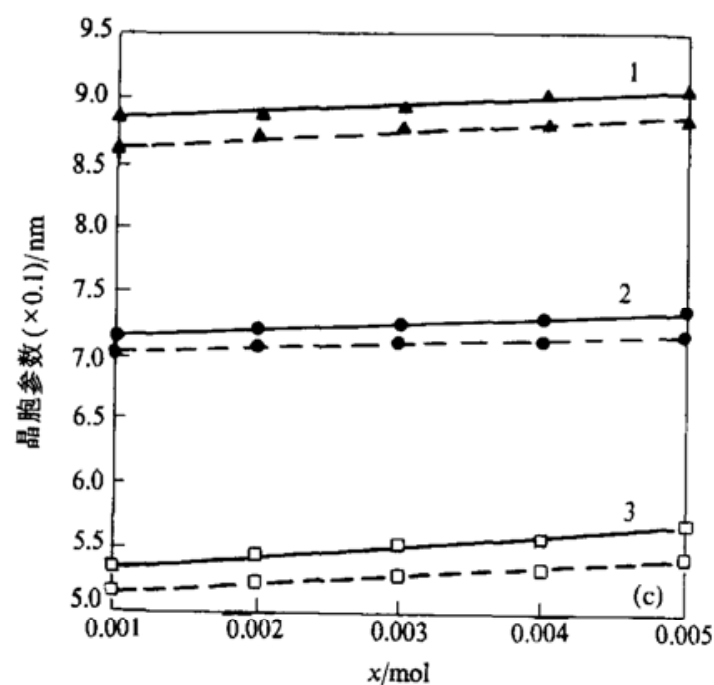

图 1 晶胞参数与钐掺杂量的关系

(1) $\mathrm{CaSO}_{4}: x \mathrm{Sm}^{3+}$; (2) $\mathrm{SrSO}_{4}: \mathrm{xSm}^{3+}$; (3) $\mathrm{BaSO}_{4}: x \mathrm{Sm}^{3+}$.

- 未辐照, -.-.--- 辐照

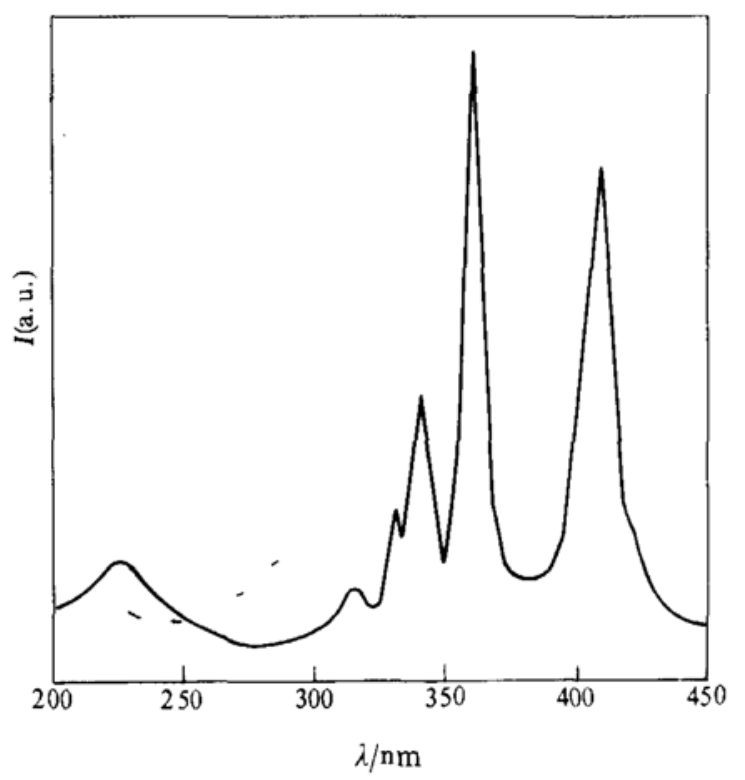

图 $3 \mathrm{MSO}_{4}: 0.002 \mathrm{Sm}^{3+}$ 的激发光谱 $(\mathrm{M}=\mathrm{Ca}, \mathrm{Sr}, \mathrm{Ba})$

$\lambda_{\mathrm{mm}}=604 \mathrm{~nm}$ 
根据 Scherrer 公式求得发光体的晶粒大小为 $8 \sim 20 \mathrm{~nm}$, 表明制得的粉料为超微细粉.

\section{$2.2 \mathrm{MSO}_{4}: x \mathrm{Sm}^{3+}$ 的荧光光谱特征}

图 2 为发光体 $\mathrm{MSO}_{4}: x \mathrm{Sm}^{3+}(\mathrm{M}=\mathrm{Ca}, \mathrm{Sr}, \mathrm{Ba}, x=0.002)$ 的发射光谱 (其余样品均与此相 似). 图中观察到了 $\mathrm{Sm}^{3+}$ 离子的 ${ }^{4} G_{5 / 2} \sim{ }^{5} H_{J}(J=5 / 2,7 / 2,9 / 2,11 / 2)$ 四条 $f-f$ 跃迁发射. 由图 还可知, 随着 $\mathrm{M}^{2+}$ 离子的电负性, 半径的变化, 导致 $\mathrm{Sm}^{3+}$ 能级的擘裂和晶场对称性变化, 从而 影响其发光强度的变化, 即在对称性低的 $\mathrm{CaSO}_{4}: x \mathrm{Sm}^{3+}$ 发光体中发光强度变大.

图 3 为 $\mathrm{MSO}_{4}: x \mathrm{Sm}^{3+}(x=0.002)$ 的激发光谱. 可以看出, 它是由两种形式的吸收峰构成, 一 种是对应于 $\mathrm{Sm}^{3+}$ 离子的四条 $f-f$ 跃迁的一系列锐线吸收, 另一种是位于 $230 \mathrm{~nm}$ 左右能量较高 的对应于 $\mathrm{Sm}^{3+}$ 离子与配体之间电荷迁移的宽吸收带. 实验结果还表明, 随着 $\mathrm{M}^{2+}$ 由 $\mathrm{Ca}$ 到 $\mathrm{Ba}$ 的变化, $\mathrm{Sm}^{3+}$ 离子电荷迁移激发带的最大位移向长波方向移动, 分别为 $226 \mathrm{~nm}, 229 \mathrm{~nm}$ 和 $232 \mathrm{~nm}$.

$\gamma$-射线辐照后样品的发射光谱如图 4 所示. 由图可见, 辐照后部分 $\mathrm{Sm}^{3+}$ 被还原为 $\mathrm{Sm}^{2+}$, 光 谱中出现 $\mathrm{Sm}^{2+}$ 的特征发射, 即于 $638 \mathrm{~nm}, 683 \mathrm{~nm}, 700 \mathrm{~nm}$ 和 $727 \mathrm{~nm}$ 附近分别呈现出 $\mathrm{Sm}^{2+}$ 离子 的 ${ }^{5} D_{0} \sim{ }^{7} F_{J}(J=0,1,2)$ 跃迁发射. 根据 Sriniras 等人间理论, 我们认为, 样品经 $\gamma$-射线辐照 后, 部分氧原子被激励放出一个电子, $\mathrm{Sm}^{3+}$ 离子捕获这个电子而被还原为 $\mathrm{Sm}^{2+}$ 离子, 从而在发 射光谱中同时出现 $\mathrm{Sm}^{3+}, \mathrm{Sm}^{2+}$ 特征发射.

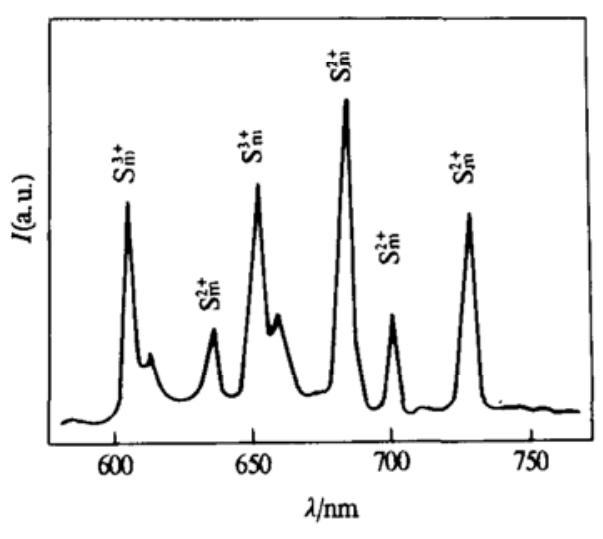

图 $4 \mathrm{BaSO}_{4}: 0.002 \mathrm{Sm}^{3+}$ 辐照后的发射光谱 $\lambda_{\alpha}=408 \mathrm{~nm}$

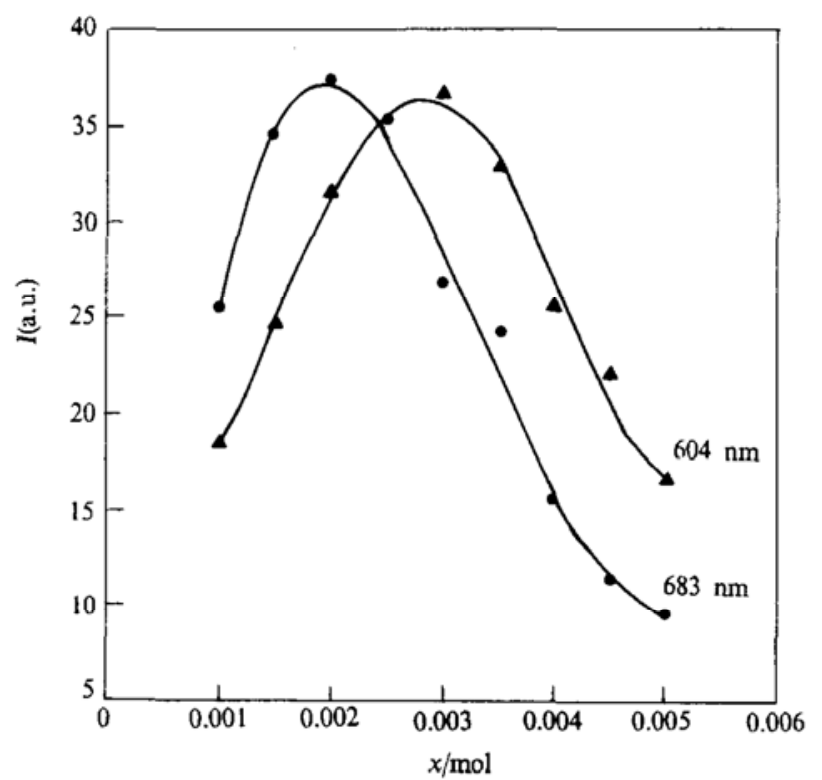

图 $5 \mathrm{BaSO}_{4}: x \mathrm{Sm}^{3+}$ 的浓度猝灭曲线 $\mathrm{Sm}^{3+}: 604 \mathrm{~nm} ; \mathrm{Sm}^{2+}: 683 \mathrm{~nm}$

发光体中中心离子的浓度与相对发光强度关系如图 5 所示. 未辐照的发光体, 当 $\mathrm{Sm}^{3+}$ 离 子浓度 $x \approx 0.003$ 基质时, 发光强度最大, 被辐照的发光体, 当 $\mathrm{Sm}^{3+}$ 离子浓度 $x \approx 0.002$ 基质 时, $\mathrm{Sm}^{2+}$ 离子的发光强度为最大. 继续增大钐离子浓度时, 则发生浓度猝灭. 根据 Ozawa 等 人门理论, 求得 $\theta=10$ 和 8 . 说明发光体辐照前后浓度猝灭分别是电四极 - 电四极相互作用和电 偶极 - 电四极相互作用引起的. 


\section{参考文献}

1 Calvert R L. Thermoluminescence and Radiophotoluminescence from Eu-and Sm-doped CaSO 4 . Phys Stat Sol (A), 1984, 83: $597 \sim 604$

2 Winnacker A. Photon-gated hole burning: a new mechanism using two-step photoionization. Optics Letters, 1985, 10(7): $350 \sim 352$

3 Lindmayer J. Electronic optical-storage technology approaches development phase. Laser Focus World, 1989, $119 \sim .127$

4 Manthey W J. Crytal field and site symmetry of trivalent cerium ions in $\mathrm{CaF}_{2}$ : the $\mathrm{C}_{4 v}$ and $\mathrm{C}_{3 v}$ centers with interstitalfluoride charge compensator. Physical Review B, 1973, 8(9): $4086 \sim 4098$

5 鲁尔兵. $\mathrm{CaF}_{2}: \mathrm{Ce}^{3+}$ 晶体的光谱性质及 $\gamma$ 线辐照效应. 科学通报, 1992, 37(5): $418 \sim 420$

6 Sriniras B. The gamma-ray induced chemisorption of oxygen on perovkite type catalysts: determination by reduction with hydrazine sulphate/hydroxylamine hydrochloride. J Radioanal Nucl Chem Letters, 1986, 107(4): $225 \sim 238$

7 Ozawa L. Themechamism of the emission color shift with activator concentration in $\mathrm{Eu}^{3+}$ activated phosphors. J Electrochem, 1971, 118(10): $1678 \sim 1679$ 